\title{
Novel Coronavirus: What to Learn from a Microbiologist?
}

\author{
Deepinder K. Chhina ${ }^{1} \quad$ Jyoti Chaudhary ${ }^{1}$ \\ ${ }^{1}$ Department of Microbiology, Dayanand Medical College \& \\ Hospital, Ludhiana, Punjab, India
}

\begin{abstract}
Address for correspondence Deepinder K. Chhina, MD, Department of Microbiology, Dayanand Medical College \& Hospital, Tagore Nagar, Civil Lines, Ludhiana 141001, Punjab, India (e-mail: Deepinder.chhina@rediffmail.com).
\end{abstract}

Ind J Car Dis Wom:2020;5:282-284

\begin{abstract}
Keywords

- SARS-CoV-2

- pandemic

- RTPCR

- antigen test

- ELISA

In December 2019, an outbreak of coronavirus disease 2019 (COVID-19) caused by SARS-CoV-2 (severe acute respiratory syndrome coronavirus 2) was declared in Wuhan, city of China. Later on, the World Health Organization declared COVID-19 as a pandemic on March 11, 2020. The clinical course of the disease ranges from asymptomatic cases to fatal severe respiratory illness. Various laboratory tests are available for the diagnosis of disease. The gold standard test is real-time PCR (polymerase chain reaction), whereas antigen testing can be used as a point-of-care test. Antibody detection by ELISA (enzyme-linked immunosorbent assay) can be used for the surveillance of the immune response.
\end{abstract}

\section{Introduction}

The coronavirus came into light in December 2019 when China reported the World Health Organization (WHO) about a cluster of pneumonia cases of unknown cause in Wuhan City in Hubei Province. The causative agent of the disease was identified as a new virus and named severe acute respiratory syndrome coronavirus 2 (SARS-CoV-2), and the disease was named as coronavirus disease 2019 (COVID-19). Within a month, the disease spread in other parts of China and worldwide. On January 30, 2020, COVID-19 was officially declared as a public health emergency of international concern by the WHO. As of June 28, 2020, a total of 9,825,539 confirmed cases globally, including 495,388 deaths, were reported to the WHO. ${ }^{1}$ In our laboratory, from the month of April to June, a positivity rate of $2.8 \%$ was seen (3\% among females and $2.7 \%$ among males). No significant difference was seen between males and females. Positivity is seen more in asymptomatic cases (3.6\%) than symptomatic cases (1.3\%).

Coronavirus is a group of viruses that are enveloped, nonsegmented, positive-sense RNA viruses showing crownlike appearance under electron microscope. Seven coronavirus can cause human disease. Alpha coronavirus (229 E, NL63) and betacoronavirus (HKU1, OC43) cause mild respiratory disease in humans. The severe acute respiratory syndrome (SARS in 2003) and Middle East respiratory syndrome (MERS in 2010) outbreaks were caused by betacoronavirus. Now, SARSCoV- 2 is a new betacoronavirus causing severe respiratory illness having genome identical to bat coronavirus. The virus might be transmitted from bats to humans through an unknown intermediate host. Human-to-human transmission of virus occurs due to contact with patients or carriers. As with other respiratory pathogens, the transmission occurs through respiratory droplets (particles $>5-10 \mu \mathrm{m}$ in diameter) by coughing and sneezing and also through hands by touching the contaminated surfaces. The spread primarily occurs from close contacts ( $<6$ feet), mainly from family members and health care professionals. Aerosol transmission and contamination of objects and surfaces are also possible. As there is a possibility of virus transmission before symptoms and thus asymptomatic individuals could transmit the virus, isolation is the best approach to control the spread of the disease. ${ }^{2}$

Theprimary receptor for the virus is angiotensin-converting enzyme 2 (ACE-2), which is usually present on the epithelial cells, vascular epithelial cells, and macrophages in the lungs. Clinical spectrum of coronavirus infection varies from asymptomatic to respiratory failure needing mechanical ventilation to multiple organ dysfunction syndrome. The common symptoms include fever, cough, fatigue, diarrhea, and dyspnea. People with underlying diseases such as diabetes and cardiac diseases are more prone to acquire infection, and mortality is also found higher among these patients. ${ }^{3}$

The spread of SARS-CoV-2 has compelled us all to observe our hygiene habits such as what we touch and how we clean. Approximately, 1,000 bacteria per square centimeter colonize human skin, especially armpits and groin areas, and 
shed these into the surrounding environment. Aerosols are generated while sneezing and coughing or from mucous secretions. As a result, we are constantly picking up microorganisms from the external environment. Avoiding crowded places and following social distancing, cough etiquette, good hand and face hygiene practices are important defense mechanisms against the highly infectious virus. ${ }^{4}$ The importance of hand hygiene cannot be underemphasized. Handwashing is important as a routine but very important for controlling the spread of viruses during the outbreak. However, it must be done properly with soap and water. If soap and water are not available, use of alcohol-based hand sanitizer for 20 seconds is the next best option. ${ }^{5}$

In much of the world, women are traditionally responsible for domestic sanitation and maintaining a hygienic home environment. ${ }^{5}$ Women can be a role model for promoting hand hygiene and healthy habits in the family. Personal hygiene habits have far-ranging consequences. Proper handwashing not only reduces the spread of coronavirus infection but also prevents the spread of other illnesses such as flu, SARS, and diarrhea. What makes the current coronavirus outbreak scarier is the high infectivity and the large number of asymptomatic carriers. That means that anyone, even healthy individuals, may be harboring the virus and can transmit it to people they come in contact with. This reiterates the importance of social distancing, cough etiquette, hand washing, and use of face masks. ${ }^{6}$

Ever since the pandemic of the novel coronavirus landed on our doorstep, epidemiologists, public health experts, microbiologists, and research scientists have been working day and night to fight it. Microbiologists are the frontline warriors, playing a key role in the diagnosis and confirmation of clinically suspected cases. Microbiologists deal with infections all the time. They diagnose and detect infections and relay it to the physicians/surgeons. ${ }^{5}$ When needed, they isolate the infection-causing bacteria and study their response to different antibiotics for proper treatment. In the present situation, they are involved in sample collection, testing, making policies, and helping in hospital infection control, all the time staring at the risk of infection on the face. For the diagnosis of SARS-CoV infection, nasopharyngeal and oropharyngeal swabs are collected in viral transport media and transported in triple packaging under a cold chain to the laboratory. For antibody testing, blood samples are taken. Recently, a study has reported the use of noninvasive samples as saliva or gargled water samples to detect coronavirus. ${ }^{7}$ Real-time polymerase chain reaction (RTPCR) of respiratory samples is the recommended gold standard method for identification, and laboratory confirmation of SARS-CoV-2 infection requires a biosafety level 2 laboratory facility. In open RTPCR, $E$ gene and $N$ gene are detected for the screening of SARS-CoV-2, whereas $S$ gene, $R d R p$ gene, and orf gene are used for the confirmatory diagnosis of SARS-CoV-2. Open RTPCR takes 6 to 8 hours for testing of 80 to 90 samples in a lot. Closed RTPCR (GeneXpert, TrueNAT) takes 2 to 3 hours with a limitation of 2 to 16 samples tested at a time. The number of samples that can be tested depends on the module of the machine. GeneXpert (CBNAAT) is a closed confirmatory cartridge based RTPCR system for testing SARS-CoV-2, having the capacity of testing 4 to 16 samples in one batch. TrueNAT can test two to four samples in one batch; this screening test detects $\mathrm{E}$ gene, and only positive samples need to be confirmed subsequently by detecting the Rdrp gene. The new BioFire FilmArray is a closed nested multiplex RTPCR system approved by the U.S. Food and Drug Administration and the Indian Council of Medical Research (ICMR). The BioFire Respiratory 2.1 plus panel tests simultaneously 19 viruses including SARS-CoV-2 and 4 bacteria causing respiratory tract infections in 1 hour. Therefore, manual open RTPCR is a test of choice for testing a large number of samples, whereas closed RTPCR is a good option for the diagnosis of infection in emergency cases. Rapid diagnostic tests detecting the presence of viral proteins (antigens) in respiratory samples is a point-of-care test that gives result within 30 minutes, but negative results have to be reconfirmed by the gold standard RTPCR. Test for the detection of antibodies in the blood of people infected with COVID-19 are available for the surveillance of immune response and not for the diagnosis of COVID-19. ${ }^{3}$ Antibodies most commonly become detectable 1 to 3 weeks after symptom onset and last for several months. As per the Centers for Disease Control and Prevention, there is no identified advantage of performing the separate assay tests for immunoglobulin (Ig) G/IgM/or total antibody. Detection of IgG antibodies for SARS-CoV-2 may be useful in high-risk population (frontline workers, health care workers, immunocompromised people). The ICMR recommends only IgG-based CLIA (chemiluminescence enzyme immunoassays) and ELISA (enzyme-linked immunosorbent assay) assays to conduct serosurveys. ${ }^{1}$

Putting personal safety on the backburner, microbiologists are providing their laboratory skills for testing a large number of samples that need to be processed during this pandemic. With several times more chance of exposure to the virus, safety of the microbiologists and allied personnel is critical. Personal protective equipment, good laboratory safety practices, and hand hygiene are the shields. Appropriate biomedical waste management facilities are must. All COVID laboratory waste should be discarded in double-layered bags with proper seal and bar code. ${ }^{1}$

With an expertise in infectious diseases good infection control practices and diagnostic facilities, microbiologists will be able to help not only in the current crisis but also in the coming future.

Healthy diet, good sleep, and regular exercise also play a key role in maintaining overall health and immunity.

\section{Conflict of Interest}

None declared.

\section{References}

1 Centers for Disease Control and Prevention. Initial Public Health Response and Interim Clinical Guidance for the 2019 Novel Coronavirus Outbreak - United States, December 
31,2019- February 4, 2020. Available at: https://www.cdc.gov/ $\mathrm{mmwr} /$ volumes $/ 69 / \mathrm{wr} / \mathrm{mm} 6905 \mathrm{e} 1 . \mathrm{htm}$ ?s_cid=mm6905\%20 e1_w

2 World Health Organization. Pneumonia of cause - China. Available at: https://www.who.int/csr/don/05-january-2020pneumonia-of-unkown-cause-china/en/\#: : text=0n\%20 $31 \% 20$ December\%202019\%2C\%20the,the\%20national\%20 authorities\%20in\%20China.

3 World Health Organization. Coronavirus Disease (COVID-19) Dashboard. Available at: https://covid19.who.int/

4 Centers for Disease Control and Prevention. Coronavirus (COVID-19). Available at: https://www.cdc.gov/coronavirus/2019-ncov/index.html
5 Guo YR, Cao QD, Hong ZS, et al. The origin, transmission and clinical therapies on coronavirus disease 2019 (COVID-19) outbreak - an update on the status. Mil Med Res 2020;7(1):11

6 Cascella M, Rajnik M, Cuomo A, et al. Features, evaluation and treatment coronavirus (COVID-19). In: StatPearls [Internet]. Treasure Island, FL: StatPearls Publishing; 2020

7 Pasomsub E, Watcharananan SP, Boonyawat K, et al.. Saliva sample as a non-invasive specimen for the diagnosis of coronavirus disease 2019: a cross-sectional study. Clin Microbiol Infect 2020 (e-pub ahead of print). doi:10.1016/j.cmi.2020.05.001 